\title{
Enterprise Interoperability: A Standardisation View
}

\author{
David Chen ${ }^{1}$, and François B. Vernadat ${ }^{2}$ \\ ${ }^{\prime} L A P / G R A I$, University Bordeaux I, France ${ }^{2}$ MACSI-INRIA \& LGPIM, ENIM/Université de \\ Metz,France, chen@lap.u-bordeaux.fr
}

\begin{abstract}
Standards that significantly contribute to achieve enterprise interoperability regarding both intra and inter-organisational environments are identified and reviewed. The concept of interoperability is firstly clarified and defined in comparison with some adjacent notions such as portability, compatibility and integration. A brief overview of standards in enterprise modelling and engineering is then given to better state their prerequisite role to standards more directly related to enterprise interoperability discussed next. Future needs to improve interoperability are discussed as part of the conclusion.
\end{abstract}

\section{INTRODUCTION}

With the globalisation of commerce, distribution and manufacturing, cooperation between enterprises of different sectors and cultures is significantly increasing. It is not only limited to sub-contracting and co-operation with suppliers and customers better known as 'supply chain' or 'extended enterprise', but also concerned with 'virtual enterprise' that can form and dissolve very quickly. Nevertheless, globalisation does not mean unification and homogenisation. Instead, there exists a strong demand to preserve cultural identity and particular ways of working within companies. This leads inevitably to some interoperable situation rather than to tight integration. Furthermore, at a company level, the need for flexibility and reactivity, the inspiration of employees to work in a more autonomous way using their own methods and tools, oblige enterprises to change from traditional hierarchically based organisation to smaller, autonomous, distributed unity structure. 
Within this context, interoperability within and between enterprises becomes a necessity and a key success factor of competitiveness.

Without standards there will be no interoperability. The objective of the paper is to identify issues and review standards relating to enterprise interoperability in intra and inter-organisational environment. It focuses on standards in the area of manufacturing activities and is presented from the point of view of users who design and implement enterprise systems. In section 2, the paper will present some basic concepts and definitions to clarify the concept of enterprise interoperability. The link and difference between interoperability and integration are tentatively discussed. Section 3 will provide an overview on standards in the area of enterprise modelling and engineering. Although these standards do not directly deal with the interoperability issues, they contribute to improve the ability of interoperation as well. Section 4 reviews standards and projects that significantly support the achievement of enterprise interoperability. A synthesis summary will be given in the last section to conclude the paper.

\section{ENTERPRISE INTEROPERABILITY}

The term 'interoperability' is increasingly used in enterprise engineering and its related standardisation activities. Generally speaking, interoperability is a measure of the ability of performing interoperation between two different entities (be they software, processes, systems, organisations...). According to the Oxford Dictionary, interoperable means 'able to operate in conjunction'. The word "inter-operate" also implies that one system performs an operation on behalf of another system. Originally, the concept of 'interoperability' comes from software engineering. From this point of view, interoperability means that two co-operating software systems can easily work together without a particular interfacing effort. It also means establishing communication and sharing information and services between software applications regardless of hardware platform(s). In other words, it describes whether or not two pieces of software from different vendors, developed with different tools, can work together. The ISO 16100, (2000) standard defines the manufacturing software interoperability as the 'ability to share and exchange information using common syntax and semantics to meet an application-specific functional relationship through the use of a common interface'.

Interoperability is however not only concerned with software applications. It may happen between any two entities in a heterogeneous or homogenous networked environment. TOGAF (The Open Group Architecture Framework, (Open Group, 2000)) defined interoperability as: '(1) the ability 
of two or more systems or components to exchange and use shared information, and (2) the ability of systems to provide and receive services from other systems and to use the services so interchanged to enable them to operate effectively together'.

Enterprise Interoperability is therefore concerned with interoperability between organisational units or business processes either within a large distributed enterprise or within a network of enterprises (e.g. supply chain, extended enterprise or virtual enterprise).

The concept of interoperability has to do with the concept of portability. TOGAF (Open Group, 2000) has defined portability as: (1) the ease with which a system, component, data, or user, can be transferred from one hardware or software environment to another; and (2) a quality metric that can be used to measure the relative effort to transport the software for use in another environment or to convert software for use in another operating environment, hardware configuration, or software system environment. In other words, portability is the ability of data or system to be moved, and interoperability is the ability of software or systems to understand and use information coming from other software or systems. The notion of interoperability is also linked to the concept of compatibility, which is a related term, at least for entities shared or in co-operation in the interoperable environment.

The ability for different systems to work together may be characterised at various levels of co-operation (e.g. physical systems, application, business and networked organisation). Clearly, interoperability has the meaning of coexistence and co-operation, while integration relates to the notions of coordination and unification. Vernadat, (1996) defines interoperability as the ability to communicate with pier systems and access the functionality of the pier systems, while integration is a broader concept embracing communication, co-operation and co-ordination capabilities. Thus, interoperability must be achieved to achieve real integration. The difference between integration and interoperability has been further clarified in ISO 14258, (1999) - Concepts and rules for enterprise models. This standard considers that there are three ways to relate models (entities) to one another: (1) Integration: there is a standard format for all constituent systems. Diverse models are interpreted in the standard format. This format must be as rich as the constituent system models. (2) Unification: there is a common meta-level structure across constituent models, providing a means for establishing semantic equivalence. The meta-model is not in an executable entity as it is in the integrated situation but a model mapping mechanism. (3) Federation: the federated model scenario may exist if no agent successfully or globally can impose requirements for semantic equivalence across all models of an enterprise. According to ISO 14258, (1999) the federated situation is the most probable scenario for full interoperability wherein most models will not be in a standard- 
ised or common form because it is not economically feasible to put them in such a form. The advantage of the federated situation is that it not only allows to collectively provide a (complex) service, but also to preserve the independence and autonomy of its components, to be open to dynamic change in composition and distribution. In such a situation, interoperability requires that co-operating entities be dynamically accommodated rather than having a predetermined meta-model. This assumes that the concept mapping (or semantic unification) is done at an ontology level, i.e. a semantic level (ISO $14258,1999)$.

\section{ENTERPRISE MODELLING AND ENGINEERING STANDARDS}

Enterprise modelling and engineering are prerequisites to Enterprise Interoperability. This section briefly presents an overview of standards related to enterprise modelling and engineering. The main standards for this area have been developed by CEN TC310/WG1 (European Standardisation Committee), ISO TC184/SC5/WG1 and to a lesser degree OMG (Object Management Group).

Concerning enterprise modelling, one can mention:

\begin{tabular}{l|l}
\hline ENV 40003,(1990) & Modelling Framework for Enterprise Integration, \\
\hline ISO 14258,(1998) & Concepts and Rules for Enterprise Models \\
\hline ISO 15704,(1998 & Requir. for Enterprise Reference Architecture and Methodologies \\
\hline ISO/IEC 15288, (1999) & Life-Cycle Management System / Life Cycle Processes \\
\hline
\end{tabular}

These standards define concepts and principles in terms of architecture and methodology for enterprise modelling and engineering. They do not deal with the representation of how an enterprise is structured or operated.

Enterprise modelling languages that can be used within the frame of the standards mentioned above have also been a concern for standardisation. For example:

\begin{tabular}{l|l}
\hline ENV 12204,(1995) & Constructs for Enterprise Modelling \\
\hline ISO 18629,(2001) & Process Specification Language (PSL) \\
\hline ISO 10303/11,(1992) & EXPRESS \\
\hline ISO/IEC 15414,(2000) & Open Distributed Processing (ODP) - Enterprise Language \\
\hline ISO/IEC 15909,(1997) & High-level Petri nets \\
\hline
\end{tabular}

Among them, ENV 12204 and ISO 15414 support multi-view enterprise modelling. PSL, Petri nets and EXPRESS are formal languages that can be directly implemented on computers. Future work in this area is related to the initiative to develop UEML (Unified Enterprise Modelling Language) (Vernadat, 2001; IST-34229, 2001). An enterprise model built using a specific 
language (for example IDEF0) can be translated into another one (e.g. GRAI nets) via UEML constructs used as a neutral format.

Standardisation also contributes to the development of reusable models (or partial models) that represent parts of enterprise structures in terms of processes, information, resources, ... The use of these models in enterprise engineering can shorten design delays and increase modelling consistency. Main reference documents are:

\begin{tabular}{l|l}
\hline ISO TR 10314, (1991) & Reference Model for Shop Floor Production Standards \\
\hline ISA-dS95, (1999) & Enterprise-Control System Integration \\
\hline ISO 15531, (2000) & Manufacturing Management Data Exchange (MANDATE). \\
\hline
\end{tabular}

These documents mainly focus on the function and information aspects. MANDATE also deals with the resource data definitions.

Concerning application development, standards are concerned with system programming such as ISO 13281.2, (1996) - Manufacturing Automation Programming Environment (MAPLE), and OMA (Open Management Architecture) by OMG, (1992).

\section{ENTERPRISE INTEROPERABILITY STANDARDS}

Enterprise Interoperability is concerned with communication and cooperation between software components, processes, organisation units and humans. To make interoperability happen, exchange of concepts is a key issue. Thus, terminology must be agreed and semantic equivalence established.

\subsection{The terminology issue}

One of the main obstacles to interoperability arises from the fact that 'the systems that support the functions in many enterprises were created independently, and do not share the same semantics for the terminology of their process models' (Schelenoff, 2000). Without explicit definitions for the terms, it is difficult to see how concepts in each application correspond to one another. Simply sharing terminology is not sufficient to support interoperability; the applications must share their semantics, i.e. meanings of their respective terminologies. In TOGAF, it has been underlined that 'interoperability can only be achieved when information is passed, not when data is passed' (Open Group, 2001). This implies that information must be correctly interpreted and understood. 
Two projects of interest, which deal with the terminology issue to support enterprise interoperability are: (1) ISO PDTR 16668, (1999) - Basic Semantic Register (BSR) and (2) ISO CD 18629-1, (2001) - Process Specification Language (PSL). In the PSL project, it has been proposed to define a formal semantic layer (called PSL ontology). Within the PSL ontology, the semantics of terminology is specified using KIF (Knowledge Interchange Format), which is itself an ISO standard. In the BSR project a different approach is used to approach the terminology issue. It consists in providing a methodology to establish standard terms by creating semantic equivalence rather then to define the terms themselves. The project has defined three main components of BSR as: (1) Semantic Components, (2) Semantic Units and (3) Bridges.

The semantic components are Units of thought used in everyday life. They may be named by the use of single or multi word terms. They will be used to specify Semantic Units. For examples: Delivery, Actual, Latest, Person, PurchaseOrder, BillOfMaterial, Date, Identifier, etc. The Semantic Units are equivalence of semantically complete data element concepts, i.e. the property of an object class with full qualification. They are the basis for the specification of data elements in information systems. For examples: GoodsDelivery.Latest.Date, Sales.Information.Contact.Telephone.Number. Finally, the Bridges allow to establish links between a Semantic Unit and its equivalence in various directories.

\subsection{Message-based interoperability}

Interoperability requires that data stored in software system on one machine can be sent and "interpreted" by another software system on another machine and for different purposes. To make this happen, standards on message format and transfer are needed. Today, Internet technology seems to provide the best perspective to support intra and inter-enterprise interoperability. Standards relating to Internet technology are mainly de facto standards promoted by the World Wide Web Consortium (W3C). Among various approaches, XML (eXtensible Markup Language) (W3C, 1998) is the most promising. XML is considered particularly adapted for data exchange as it has the promise of making data "self describing". In other words, XML offers 'a non-proprietary and inexpensive way to promote reuse of data by providing a way to locate it (semantic search), and by providing a standard way to transform and move it between applications' (CIO, 2000). Coupled to $\mathrm{XML}$, some other $\mathrm{W} 3 \mathrm{C}$ specifications are also critical to interoperability. For instance (just to name a few): (1) SOAP (Simple Object Access Protocol) allows applications to communicate directly with each other through Internet by defining a simple, extensible message format in standard XML 
(W3C, 2000), (2) WSDL (Web Services Description Language) is an XML format for describing services accessible on the web as a set of endpoints operating on messages containing either document-oriented or procedureoriented information (W3C, 2001), (3) ebXML (Electronic Business XML Initiative) enables to exchange XML based messages through the web, (4) J2EE standard allows to benefit security features for authentication and access control in Java environments, and (5) RosettaNet defines XML based business interfaces to support interoperability among enterprises such as supply chain management.

\subsection{Manufacturing software interoperability}

According to ISO TC184/SC5/WG1, standards must define only what is necessary so that the software developer knows with what the software must work. Ideally, standards should enable interoperability and still protect innovation, efficiency of approach, and migration capability (Nell, 2001b). One standard dealing with manufacturing software interoperability is ISO DIS 16100 , (2000) - Manufacturing software capability profiling. This is an ongoing project carried out by ISO TC184/SC5/WG4. This standard considers that one of the most important aspects that makes interoperability operational is to describe precisely and concisely the capability of software. The capability is defined in terms of potential functions. The standard will specify a standard way of representing "what I do and what I need". The first part of the standard aims only at defining a framework for software interoperability. The ultimate goal of the project is to allow to: (1) select appropriate software, (2) substitute one software component with another, (3) migrate software to another platform, and (4) verify software to a capability profile. Manufacturing software packages concerned with this standard are CAED, NC programming systems, PDM (Product Data Managers), MES (Management Executive Systems), CAE (Computer-Aided Engineering) systems, etc.

Concerning application integration, standards are concerned with the IT based services, which are built upon the seven layers proposed by ISO 7498 OSI model (ISO/IEC 7498-1, 1994). Generally speaking, these services contribute to improve software interoperability. The main approaches are:

\begin{tabular}{l|l}
\hline ENV 13550, (1995) & Enterprise Model Execution and Integration Services (EMEIS) \\
\hline ISO 15414, (2000) & Inform. Technology - Open Distributed Processing - Ref. Model \\
\hline ISO 13281.2, (1996) & Manufacturing Automation Programming Environment (MAPLE) \\
\hline TOGAF (Open & Technical Reference Model \\
Group, 2000) & \\
\hline OMA (OMG, 1992) & Open Management Architecture \\
\hline OAGIS (OAG, 2001) & Open Applications Group Integration Specification \\
\hline
\end{tabular}


According to its developers, OAGIS better supports inter-enterprise business application interoperations, i.e. communication and interaction between companies. In other words, OAGIS is a horizontally based approach that is applicable across industry sectors. Today, OAGIS covers financial transactions, ERP to ERP interoperation, Supply Chain management, etc. On the other side, the reference model of OMA better known as CORBA (OMG, 1992), focuses on intra-enterprise interoperations of software objects that share a same infrastructure.

\subsection{Manufacturing and business process interoperability}

The ISO CD 18629-1, (2001) - Process Specification Language (PSL) originates from a NIST project aiming at developing a formal language to facilitate process interoperability (Schelenoff, 2000). The goal of PSL project is to deal with the translation problem between two inter-operable process applications. The project has considered that when the interoperation is limited to a small number of processes, the translation can be done at a oneto-one basis. However, when interoperation becomes generalised and widely applied, the development of translators between native formats of those applications and PSL can facilitate the interoperability. PSL will act as an intermediate neutral format. The view hold by PSL is that using this intermediate format can reduce the number of translators from $n(n-1)$ to $n$ in the case where there are potentially $\mathrm{n}$ processes that can enter into interoperation. This point of view is also shared in some related domains, for example: (1) ISO 10313-1, (1993) known as STEP in product data modelling, and (2) the UEML initiative in enterprise modelling to develop a Unified Enterprise Modelling Language (Vernadat, 2001).

ISO TC184/SC5/WG1 has also considered a potential standardisation effort to deal with process interoperability focusing on the definition of interaction scenario. The project is an ISO New Working Item (NWI) entitled "Rules for manufacturing process interoperability". It intends to specify the mechanism (processes and meta data) that an enterprise can use to represent and present, in a standard way, information required to establish communications for enterprise-process-interoperability (Nell, 2001a).

\section{CONCLUDING REMARKS}

To meet new industrial challenges, there is a shift from the paradigm of total integration to that of interoperation, which holds the promise for more flexibility. Relevant standardisation activities focusing on interoperability have just started and most work remains to be done. It has been found that 
Internet-based technology standards play an important role to move and transfer data/messages more easily. These approaches have been mainly developed by non-institutional organisations such as OMG, W3C and OAG, and remain de facto standards. Standards elaborated by ISO and CEN have focused more on modelling aspects dealing with the specifications of resources (e.g. software profile) and processes (PSL) as well as their related semantic and syntax problems. One of the issues for the future is to establish the link between these two communities and to map these standards to a consistent framework.

Few standards exist that directly relate to Enterprise Interoperability per se. However, it is important to note that interoperability is not only a problem of technology. It implies a better and mutual understanding between partners involved in the interoperation. Cultural inertia will limit the effectiveness and use of standards to design interoperable systems. Consequently, an entity (be it a company or a department within a company) must actively engage to a self-adapting process in terms of working procedures and culture so as to facilitate maximum exchanges of information with the outside world.

\section{REFERENCES}

Chen, D. Vernadat, F. (2001), Standardisation on Enterprise Modelling and Integration: Achievements, On-going works and future perspectives, In Proc. of IFAC 10th Symposium on Information Control in Manufacturing (Invited session), Vienna, September 20-22.

ClO Council, (2000), Enterprise Interoperability and Emerging Information Technology (EIEIT) Committee, www.cio.gov/Documents/cio_eieit_xml_workgroup_jul_2000.html.

ENV 12204, (1995), Advanced Manufacturing Technology - Systems Architecture - Constructs for Enterprise Modelling, CEN TC' 310 WG1.

ENV 13550, (1995), Enterprise Model Execution and Integration Services (EMEIS), CEN, TC 310 WG1.

ENV 40003, (1990), Computer Integrated Manufacturing - CIM systems architecture framework for modelling, CEN, TC 310 WG1.

ISA-DS95, (1999), Enterprise-Control-System Integration, ISA DS95.01-1999, Instrument Society of America.

ISO 10303-1, (1993), Industrial automation systems and integration - Product data representation and exchange - Part 1: Overview and Fundamental Principles. TC 184 SC5 WG1.

ISO 14258, (1999), Industrial Automation Systems - Concepts and Rules for Enterprise Mod$e l s$, TC 184 SC5 WG1, April-14 version.

ISO 15704, (1998), Requirements for Enterprise Reference Architecture and Methodologies, TC 184 SC5 WG1, N423.

ISO CD 18629-1, (2001), Industrial automation systems and integration, Process Specification Language (PSL), Part 1: Overview and Basic Principles, TC 184 SC4/SC5 JW8.

ISO DIS 10303-11, (1992), The EXPRESS Language Reference Manual, TC 184 SC4 WG5, N35. 
ISO DIS 13281.2, (1996), Industrial Automation Systems - Manufacturing Automation Programming Environment (MAPLE) - Functional architecture. TC 184

ISO DIS 15531-1, (2000), Industrial automation systems and integration-Manufacturing management data exchange - Part 1: Overview and fundamental principles, TC184 SC4 WG8, N138 R3.1.

ISO DIS 16100, (2000), Manufacturing Software Capability Profiling, Part 1 - Framework for interoperability, TC 184 SC5, ICS 25.040.01.

ISO PDTR 16668, (1999), Basic Semantic Register (BSR) - Rules, Guidelines and Methodology, TC 154 WG1, N007.

ISO TR 10314, (1991), Reference Model for Shop Floor Production Standards, Part 1 - Reference model for standardisation, methodology for identification of requirements.

ISO/IEC 10746-3, (1994), Information Technology - Open Distributed Processing - Reference Model - Architecture, ITU-T Recommendation X.903.

ISO/IEC 15414, (2000), Information Technology - Open Distributed Processing - Reference Model - Enterprise Language, ITU-T Recommendation X.911, Version 303, ISO/IEC JTC 1 SC 7 WG 17.

ISO/IEC 7498-1, (1994), Information Processing Systems, Open System Interconnection (OSI) Reference Model, The Basic Model, ITU-T Rec. X.200 (1994 E).

ISO/IEC CD 15288, (1999), Life-cycle management System, Life Cycle Processes, ISO JTC 1 SC 7, N2184.

ISO/IEC CD 15909, (1997), High-level Petri Nets - Concepts, definitions and graphical notations, Committee Draft, October, Version 3.4.

IST - 2001 - 34229, (2002-08-12), Unified Enterprise Modelling Language (UEML), Thematic Network, Annex 1, Description of Works, EC IST Project.

Kosanke K. Nell J.G. (Eds.), (1997), Enterprise Engineering and Integration: Building International Consensus, Springer-Verlag, Berlin, pp. 613-623.

Nell, J.G. (2001 a), Requirements for establishing manufacturing-enterprise-process interoperability, TC 184 SC5 WG1 New-work-item proposal, N433 rev.2.

Nell, J.G. (2001b), Enterprise Representation: A different paradigm for designing processinteroperability standards, TC 184 SC5 WG1, NIST, Gaithersburg, MD, USA.

OAG, (2001), OAGIS: Open Applications Group Integration Specification, Open Application Group, Incorporated, Release 7.2.1, Doc. No. 20011031.

OMG, (1992), Object Management Architecture, Version 2, Open Management Group.

Open Group, (2000), TOGAF: The Open Group Architecture Framework, Document No. 1910, Version 6.

Schelenoff, G. Gruninger, M. Tissot, F. Valois, J. Lubell, J. Lee, J. (2000), The Process Specification Language (PSL): Overview and Version 1.0 Specification, NIST, Gaithersburg, MD, USA.

Vernadat, F. (2001), UEML: Towards a Unified Enterprise Modelling Language. Proc. $3^{\text {ème }}$ Conférence Francophone de Modélisation et Simulation (MOSIM'01), Troyes, France, 2527 April, pp. 3-13. (Invited paper).

Vernadat, F.B. (1996), Enterprise Modelling and Integration: Principles and Applications, Chapman \& Hall, London.

W3C, (1998), XML, Extensible Mark-up Language, W3C XML 1.0, February.

W3C, (2000), Simple Object Access Protocol (SOAP) 1.1, W3C Note, http://www.w3.org/TR/2000/NOTE-SOAP-20000508/, May.

W3C, (2001), Web Services Description Language (WSDL) 1.1, W3C Note 15 March, http://www.w3.org/TR/2001/NOTE-wsdl-20010315. 